\title{
The development of chemical pathology ${ }^{1}$
}

\author{
ARTHUR JORDAN
}

I see the development of chemical pathology in Great Britain in two phases, the first up to the end of the second world war, and the second since that time.

In the earlier phase the emphasis was on the word chemical. The chemical pathologist was above all an analyst and this was the golden age for the development of new methods based on the traditional techniques of the chemist. The medical consequences of the results were not always well known or appreciated and so there was a tendency to regard a medical qualification as much less important than a scientific qualification in this branch of hospital laboratory work. This view is still held in some circles. I do not share it.

Since the war, the outlook has been changing and now the emphasis is increasingly on the word 'pathology'. The chemical pathologist must be first and foremost medically qualified, that is to say, he must be a clinical pathologist, as one of his important duties is to help both in diagnosis and in treatment. A number of developments have contributed to this change of outlook. The first of these is the considerable extension of our knowledge of human biochemistry, as this is changing the face of medicine. The physician needs to know not only what is happening, but why, and this often leads to a biochemical explanation so that treatment is becoming daily less empirical. Therefore if pathologists are to have a place as doctors and not as technicians only it must be because they can bring to the problems of medicine a scientific knowledge more extensive than that which clinicians have the time to acquire. In my view the primary examination for membership of our College could well require biochemistry at a level intermediate between that of the undergraduate medical curriculum and that of an honours B.Sc. school, and this without regard to the branches of pathology that the candidate was offering.

Another development has been the introduction of techniques which belong to physics rather than to chemistry. An obvious example is flame photometry. Physical methods are creeping in and these are usually quicker and more accurate.

A third development I wish to mention is auto-

'An abridged version of the Presidential address to the Association of Clinical Pathologists on 2 October 1964. mation. Much of the work in chemical pathology is $\overrightarrow{0}$ quantitative analysis. A given amount of blood orserum is subjected to a predetermined set of treat $\vec{\omega}$ ments, finishing with a reading in a photoelectrie colorimeter. Such a sequence is made for auto? mation. The advantages of automation are a pre $\vec{\infty}$ cision which is the same at 9 o'clock in the morning and at 5 o'clock at night, a machine which has no soul to be destroyed by repetitive work, and the possibility of carrying out numbers of requests more rapidly than a technician could do them on the bench. The disadvantages of automation are the need for very careful control lest the machine introduce its own errors into a whole batch of determinations, and the need for somebody with a fair degree of mechanical skill to be constantly available. A conse-quence of the introduction of automation has been a considerable loss of choice of method, but this is not entirely bad; standardized procedures make foro comparability. It is to be hoped that in future new procedures will only be adopted in routine labora $\vec{F}$ tories after much more thorough study than has been the case in the past.

Yet another post-war development in chemicap pathology has been the introduction of controls Not very long after the war a number of authors? amongst whom the late Professor King and Professor 3 Wootton deserve honourable mention, demonstrate that the same specimen sent to different laboratories for analysis evoked very different answers. This is still the case but perhaps the differences are less than? they were although the use of controls is not as general as it should be. Any laboratory that runs control series of estimations finds that there ares periods during which some particular method doesi not give the required results, although without the control the impression might be that the method was behaving satisfactorily. The laboratory needs two types of control. The first is one which permit comparison with the results of other laboratories It assesses the accuracy of the method, giving to the word 'accuracy' its statistical meaning and is best provided by one of the commercial preparations $\stackrel{\mathbb{\mathbb { C }}}{\mathrm{C}}$ Such a control is put up once or twice a week. The second type of control is one which is put up withe each batch of tests and serves as a control of pre? cision: the result should be within the controp 
limits. In our own laboratories we use batches of sterile horse serum for this control.

I think that there is still considerable scope for national surveys of the type in which the same serum (or blood) is sent to many laboratories and the results compared. I hope that a regular survey scheme may be introduced, and I should like to think that our Association might pioneer it. I hasten to add that I do not think that controls need be restricted to chemical pathology.

Two developments I must mention briefly. The increasing complexity of chemical pathology is leading to individual laboratories specializing in particular investigations. This leads to greater efficiency: no laboratory can do everything. The other development is the steady increase in the work load. In our own laboratories this increase is between $20 \%$ and $25 \%$ each year; a similar tendency is seen in all laboratories handling chemical pathological investigations.

To return to our relations with clinicians, there are a number of investigations with which the laboratory can help the physician in the field of chemical pathology which are well within the compass of the routine hospital laboratory. One of these is the diagnosis of the cause of attacks of hypoglycaemia occurring after puberty. The textbooks list a number of causes and the problem is usually to know whether the case is one of islet-cell tumour or of functional hypoglycaemia. Obviously the first question which must be asked is whether the attacks are of hypoglycaemia or no. It is only possible to be sure that attacks are, in fact, due to hypoglycaemia if a low blood sugar level is demonstrated during an attack. Hypoglycaemia itself may provoke the body to mobilize its resources and restore the blood sugar to normal and so the finding of a normal blood sugar on one or two occasions during an attack does not entirely exclude hypoglycaemia. When investigating the cause of the condition we like to have repeated fasting blood sugar levels and a six-hour glucose tolerance curve. A low fasting blood sugar level is very suggestive of an islet-cell tumour whereas functional hypoglycaemia usually shows normal fasting levels. The six-hour glucose tolerance curve is carried out exactly as is the usual glucose tolerance test but bloods continue to be taken up to six hours. A few years ago the next step might have been a fast of 48 or 72 hours for the patient, to provoke an attack if an islet-cell tumour were present. Such a fast was unpopular with the patient and not always easy to organize in the ward. Today the tolbutamide tolerance test holds out hopes of proving a useful diagnostic tool and replacing the prolonged fast. It seeks to produce hypoglycaemia and therefore intravenous glucose injections must be immediately available and the patient kept under close observation. After the tolbutamide injection the blood sugar drops, to recover to a reasonable level somewhere in the fasting range in the last one and a half hours of the test. In a case of islet-cell tumour the blood sugar level drops and recovers little, if at all, throughout the test. If the fasting level is very low, the curve remains low throughout the test. In functional hypoglycaemia, on the other hand, the tolbutamide curve remains entirely normal. These investigations are within the competence of any laboratory and will usually provide the diagnosis. Most cases of hypoglycaemia are functional hypoglycaemia but it is imperative that islet-cell tumours be diagnosed and treated.

Measuring acid-base metabolism provides another good example of a technique that any laboratory can handle. The evolution of investigations in this field is interesting. For technical reasons, determination of the blood $p \mathrm{H}$ was rarely carried out before the war, and the usual investigation was to determine the plasma bicarbonate using the Van Slyke apparatus. The result was reported in one of the numerous ways in which this particular investigation can be reported. The assumption was commonly made that any deviation of acid-base metabolism from the normal was due to non-respiratory causes. A few years ago, however, a considerable advance was made: the plasma bicarbonate and the $p \mathrm{H}$ were both determined, and this enabled one of the following diagnoses to be made: normal, respiratory acidosis, non-respiratory acidosis, respiratory alkalosis, non-respiratory alkalosis. I must emphasize that the acidosis and alkalosis relate to the blood; the situation inside the cells of the body is not necessarily the same and often we are in doubt as to what is happening intracellularly.

The next question must be the extent to which there is any compensation: for instance, non-respiratory acidosis (metabolic acidosis) might be associated with some respiratory compensation. The blood would be acid from the metabolic cause, overbreathing could occur, and the resultant loss of $\mathrm{CO}_{2}$ would lead to the blood being slightly less acid than it would otherwise be. Recognition of the importance of the $\mathrm{pCO}_{2}$ made it possible to assess the compensation. This in turn was rapidly followed by the development of the Astrup technique, which was speedily reduced to a micro method. The standard bicarbonate level is a measure of the amount of buffer in the plasma: an increase coincides with a shift of the $p \mathrm{H}$ of the blood towards the alkaline side, provided the $\mathrm{pCO}_{2}$ is held constant at the normal value of 40 . So the standard bicarbonate estimation is a measure of the non-respiratory element in the acid-base pattern. 
We have now a quantitative measure of the respiratory element and of the non-respiratory element. The respiratory element is measured by the $\mathrm{pCO}_{2}$ which will be above 40 for a respiratory acidosis and below $\mathbf{4 0}$ for a respiratory alkalosis. The nonrespiratory element is measured by the standard bicarbonate level which is less than normal in nonrespiratory acidosis and greater than the normal in non-respiratory alkalosis. Some assessment of compensation is possible.

The blood is buffered not only by the bicarbonate system but also by the haemoglobin buffer system. Another concept was therefore devised, the base excess. If the base excess is positive there is a nonrespiratory alkalosis, if the base excess is negative there is a non-respiratory acidosis. In fact, base excess or standard bicarbonate assess the same thing: the non-respiratory contribution to the acid-base balance.

Interpretation of the Astrup results is thus straightforward. For respiratory acidosis the $p \mathrm{H}$ is acid and the $\mathrm{pCO}_{2}$ is raised. This will either be uncompensated, when the base will be about zero, or partially compensated, when the base excess will be positive. Complete compensation (which does occur) would give a $p \mathrm{H}$ within the normal range, a raised $\mathrm{pCO}_{2}$ level, and a raised base excess. For non-respiratory acidosis the $p \mathrm{H}$ is acid and the base excess is negative. If partially compensated the $\mathrm{pCO}_{2}$ will be less than $40 \mathrm{~mm}$. Hg, if uncompensated it will be 40 . Thus for respiratory and non-respiratory alkalosis we shall have appropriate patterns.

The latest development in the acid base story has been to plot the actual concentration of hydrogen ions (on a logarithmic scale) against the $\mathrm{pCO}_{2}$ (still on a logarithmic scale). The whole thing is now quantitative with the two elements, respiratory and non-respiratory, expressed in the same units. If we all went over to this system most people could learn $\stackrel{0}{=}$ about the clinical applications of acid-base deter-o minations directly without needing to struggle for $\Rightarrow$ weeks to learn the terminology. It shows the end $\stackrel{\text { की }}{+}$ towards which we should all strive: simplicity.

Investigations which are simple may be over-음 looked. One of these is paper electrophoresis of urinary proteins. In the early days of chemical pathology it was recognized that albumin or globulines might appear in the urine but that the albumin- $\overrightarrow{0}$ globulin ratio from the salting-out procedure gavevery variable findings. Paper electrophoresis of thew urinary proteins, if necessary after concentration of the urine, has shown that whereas most cases of proteinuria show a preponderance of albumin and $\dot{\phi}_{\infty}$ a pattern of globulins not unlike that of serum, $\omega$ patients with a tubular lesion secrete a large amount of $\alpha_{2}$-globulin, the band of which may exceed that of the albumin in intensity. Again, in myelomatosis if there is any proteinuria, however slight, the urinary $\overrightarrow{\vec{z}}$ protein should be examined by paper electrophoresis because, although true Bence-Jones proteinuria occurs in a minority of cases of myelomatosis, an abnormal urinary protein, running in the globuliner area, is much commoner and may be present wheno the serum protein pattern is normal and therefores be of diagnostic value.

I have mentioned these three examples only; the list could be a very long one. My object has been to show that the approach of the chemical pathologist is that of a medical man approaching a medica 5 problem: the procedures are simple and their interpretation is simple. I am convinced that chemicab pathology must develop as a branch of medicines. if it develops as a branch of biochemistry, the patient will suffer and this is one of the stronges reasons for calling the subject chemical pathology and eschewing the term clinical biochemistry. 